
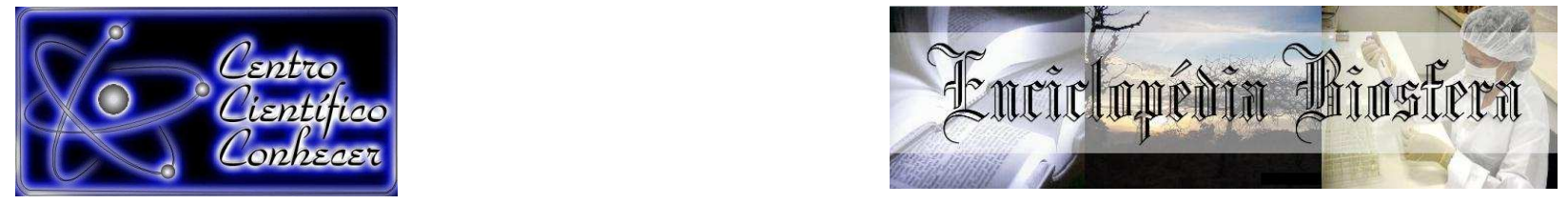

\title{
AVALIAÇÃO DO CRESCIMENTO DA CASTANHA-DO-BRASIL (Bertholletia excelsa Bonpl.) EM UM PLANTIO NO MUNÍCIPIO DE MACHADINHO DO OESTE - RONDÔNIA
}

Marilia Locatelli' ${ }^{1}$, Paulo Humberto Marcante'2, Henrique Nery Cipriani ${ }^{3}$, Eugênio Pacelli Martins ${ }^{4}$, Abadio Hermes Vieira ${ }^{3}$

1 Pesquisadora da Embrapa Rondônia e Professora do Mestrado em Geografia da Universidade Federal de Rondônia, Porto Velho, RO, marilia.locatelli@embrapa.br

2Engenheiro Florestal, Embrapa Rondônia, Porto Velho, RO, paulo.marcante@embrapa.br

3Pesquisador da Embrapa Rondônia, Porto Velho, RO, henrique.cipriani@embrapa.br, abadio.vieira@embrapa.br

4Professor do Curso de Engenharia Florestal da Faculdade de Rondônia- FARO, pacellimar@yahoo.com.br

Recebido em: 08/09/2015 - Aprovado em: 14/11/2015 - Publicado em: 01/12/2015 DOI: http://dx.doi.org/10.18677/Enciclopedia_Biosfera_2015_099

\begin{abstract}
RESUMO
A castanha-do-brasil (Bertholletia excelsa Bonpl.) é uma espécie que se destaca na região amazônica, por ter uma importante função econômica, ecológica e social. A espécie tem grande potencial silvicultural para reflorestamentos com fins madeireiros ou para produção de frutos, também podendo ser considerada apta a compor sistemas agroflorestais. O presente estudo teve como objetivo avaliar o crescimento da castanha-do-Brasil em dois sistemas de plantio, castanha consorciada com cupuaçu e castanha solteira, no município de Machadinho do Oeste - Rondônia. A área do experimento foi monitorada através de coleta periódicas de dados dendrométricos ao longo do tempo, desde sua implantação em 1987, tomando-se como parâmetros a altura total e o diâmetro a altura do peito (DAP). Pelas análises dos dados, observou-se ao longo dos 28 anos de avaliação, que o crescimento foi satisfatório em DAP, altura e volume, e que na projeção de volume futuro chegou-se a um volume de $331,81 \mathrm{~m}^{3} / \mathrm{ha}$ aos 40 anos de idade, sendo indicada para plantios em áreas degradadas, como elemento da recomposição florestal.
\end{abstract}

PALAVRAS- CHAVE: castanha do brasil, crescimento, região amazônica, volume.

\section{EVALUATION OF BRAZIL NUT (Bertholletia excelsa Bonpl.) GROWTH PLANTING IN MACHADINHO D'OESTE- RONDONIA}

\begin{abstract}
The Brazil nut (Bertholletia excelsa Bonpl.) is a species that stands out in the Amazon region, and have an important economic role, ecological and social. The species has great potential for reforestation with timber silvicultural purposes or for fruit production, and it can also be considered able to compose agroforestry. This
\end{abstract}


study aimed to evaluate the growth of the Brazil nut in two planting systems, consortium with cupuaçu and single plantation in Machadinho d'Oeste - Rondonia. The area of the experiment was monitored through periodic dendrometric data collection over time, since its implementation in 1987, taking as parameters total height and diameter at breast height - DBH. After the analyzes, it was observed over the 28 years of measurements, that growth was satisfactory in $\mathrm{DBH}$, height and volume, and the projection of future volume came to a volume of 331.81 cubic meters / ha to 40 years old and was recommended for planting in degraded areas, as part of forest replanting.

KEYWORDS: Brazil nut, growth, amazon region, volume

\section{INTRODUÇÃO}

A floresta amazônica possui uma das mais diversificadas floras do planeta com um grande número de essências florestais de interesse para o homem (HOMMA, 2014). Dentre esse grande número de espécies florestais a Bertholletia excelsa (Bonpl.) se destaca por sua importância econômica, ecológica e social (SILVA, 2012). É uma espécie que já sofreu e ainda sofre com o desmatamento, sendo que os exemplares de ocorrência natural são legalmente protegidos e considerados imunes de corte em todo o território brasileiro conforme o Decreto 1282 de 19/10/1994.

Bertholletia excelsa (Bonpl.) é uma essência florestal nativa da região amazônica, sendo uma das mais importantes espécies de exploração extrativista, pois suas amêndoas apresentam alto valor econômico, tornando-se uma das principais atividades econômicas na região amazônica (BAYMA et al, 2014). É uma espécie imponente, sendo árvore de grande porte, com uma copa grande e emergente, seu fuste é bem retilíneo sem presença de galhos, sofre desrama natural de galhos, formando um eixo ortotrópico com características madeireiras de grande interesse para a indústria. Geralmente as castanheiras são encontradas em grupos, formando os conhecidos "castanhais" (SILVA, 2014). Sua madeira é considerada ótima, podendo ser usada na construção civil e também construções navais, além dos usos como esteios e obras em ambientes externos. A espécie pode ser considerada como de grande potencial silvicultural para reflorestamentos com fins madeireiros ou para produção de frutos (HOMMA et al, 2014). Para alguns estudos feitos com castanha do Brasil na região de Manaus observou-se que ocorreu crescimento significativos em altura, DAP e para as variáveis morfométricas, denotando o potencial silvicultural da espécie, também apta a compor sistemas agroflorestais e componente importante na recuperação de áreas abandonadas e degradadas (NUNES \& VIVAN, 2011).

Para que se possa definir uma espécie para reflorestamento são necessários observar criteriosamente vários aspectos, como sua suscetibilidade a doenças e pragas quando em plantios homogêneos, sua aptidão em relação ao sítio e sua capacidade produtiva, qualidade da madeira, capacidade de produção de frutos comerciáveis, demanda do mercado pela madeira e pelos frutos (SABOGAL et al, 2006). No Brasil a escolha de espécies nativas para reflorestamento apresenta inúmeras dificuldades, e talvez a que mais limite as decisões de escolha seja a escassez de informações das espécies para definir o potencial silvicultural, por isso se faz necessário estudos da potencialidade de crescimento de algumas espécies nativas, em determinados sítios, para que se tenha subsídios na hora de definir qual a melhor espécie a ser escolhida. 
Com base no exposto esse trabalho teve como objetivo a prognose de volume aos 40 anos, para um plantio de castanha do Brasil, localizado no município de Machadinho do Oeste - RO.

\section{MATERIAL E MÉTODOS}

\section{Local e caracterização da área de estudo}

A área de estudo localiza-se no campo experimental da Empresa Brasileira de Pesquisa Agropecuária (EMBRAPA) Rondônia, na RO 133, Km 3, em Machadinho d'Oeste, Rondônia. O local está situado sob as coordenadas 9²3'49" latitude sul, 6201'15" longitude oeste Gr. E altitude $130 \mathrm{~m}$, a uma distância de $350 \mathrm{~km}$ da capital Porto Velho. O campo possui uma área total de 219,10 ha, sendo que 184,10 ha de floresta ombrófila densa e 25,00 ha de áreas abertas para experimentos diversos e infraestrutura, com latossolo amarelo e textura argilosa, o relevo é plano (Figuras $1 \mathrm{e}$ 2).

O tipo climático segundo a classificação de Köppen é Am, caracterizando-se como megatérmico (temperatura média do mês mais frio superior a $18^{\circ} \mathrm{C}$ ). A estação chuvosa vai de dezembro a março, com $1.300 \mathrm{~mm}$, e um total anual de precipitação de $2.390 \mathrm{~mm}$, e uma estação seca bem definida nos meses de junho e julho. Segundo dados de série histórica, a temperatura média anual é em torno de $26,2{ }^{\circ} \mathrm{C}$, sendo que a média das temperaturas máximas é de $31,5 \stackrel{\circ}{\circ}$, a média das temperaturas mínimas é de $20,9 \stackrel{\circ}{\circ}$. A média da umidade relativa do ar é de $85 \%$, apresentando pouca variação durante o ano, de 80 - 89\% (INMET, 2015).

Para o estudo foi utilizado um experimento implantado no campo experimental, com delineamento experimental em blocos com dois tratamentos, um com plantio de castanha do brasil em consórcio com cupuaçu e outro com plantio apenas da castanha do brasil, tendo cada tratamento quatro repetições. Para a castanha do brasil, nos dois tratamentos foi utilizado um espaçamento de $12 \mathrm{~m} \times$ $12 \mathrm{~m}$, e para o plantio do cupuaçu foi utilizado o espaçamento de $6 \mathrm{~m} \times 6 \mathrm{~m}$. Em cada bloco foram plantadas 25 plantas de castanha do brasil, totalizando 200 plantas, sendo 100 plantas em cada tratamento.

A implantação do experimento foi realizada em fevereiro de 1987, e o local onde foi instalado era uma floresta, que foi derrubada e queimada, e os restos não queimados foram encoivarados e novamente queimados e realizado o rebaixamento dos tocos. O plantio foi realizado através de abertura de covas, e na ocasião do plantio foi adicionada adubação de 100 gramas de superfosfato triplo nas covas, tanto para castanha quanto para o cupuaçu. Nos dois primeiros anos do sistema, 1987 e 1988, foi cultivado nas entrelinhas do plantio, arroz e feijão caupi, respectivamente. Para os tratos culturais foram apenas realizadas limpezas mecânicas, através de roço com trator. 


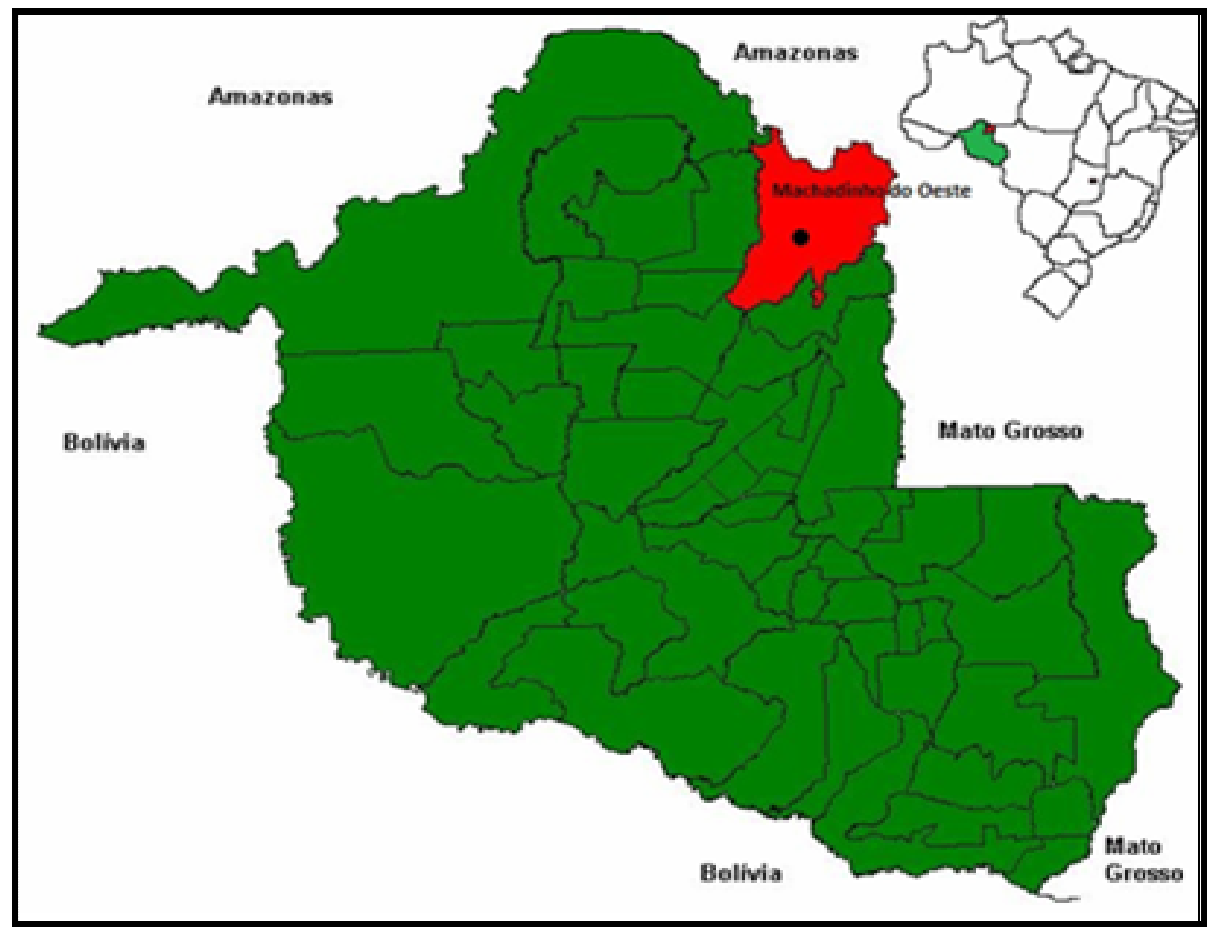

FIGURA 1 Localização da cidade de Machadinho do Oeste - RO.

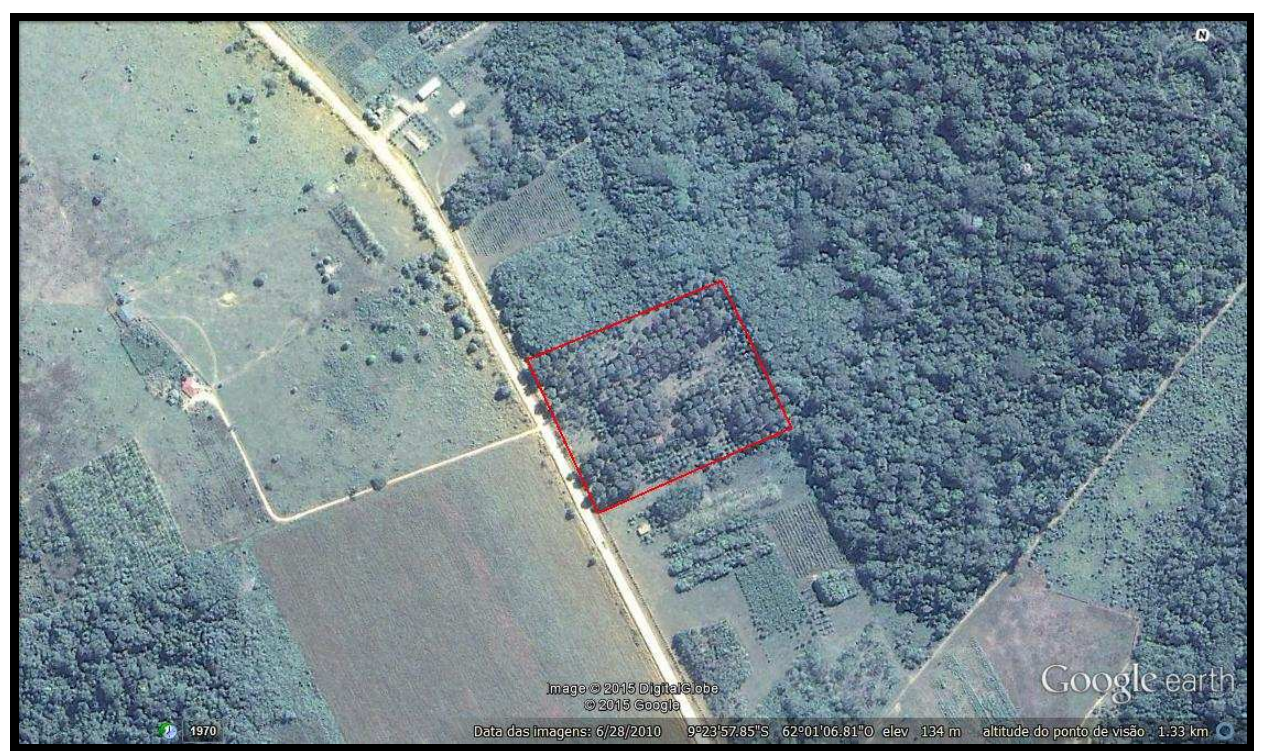

FIGURA 2 Área do experimento e plantio no Campo Experimental da EMBRAPA em Machadinho do Oeste - RO.

\section{Avaliações dendrométricas}

A área do experimento foi monitorada através de coleta periódica de dados dendrométricos, desde sua implantação em 1987, tomando-se como parâmetros a altura total $(\mathrm{Ht})$, através de hipsômetro. As tomadas de diâmetro a altura do peito (DAP, 1,30 m do chão) foram realizadas com fita diamétrica, a partir de 1990 quando as plantas já apresentavam altura suficiente para a tomada dessa medida. Com o levantamento obteve-se o diâmetro médio de cada tratamento, para comparação de 
desempenho, e em 2010 além da altura total, foram tomadas as alturas comerciais até a altura da inserção dos galhos da copa, com hipsômetro eletrônico Vertex®.

Através do DAP calculou-se a área transversal pela equação 1, e com os resultados foi realizada a comparação de desempenho entre os dois tratamentos, castanha solteira e castanha consorciada.

Equação 1.

$$
g=\frac{\pi D a p^{2}}{40000}
$$

Também foram calculados a área basal por ha e o volume por ha.

Para determinação de volume utilizou-se a formula de volume tradicional, conforme equação 2, considerando um valor de 0,7 para o fator de forma, visto que não foi determinado um fator de forma específico para o povoamento.

Equação 2.

$$
V=\frac{\pi D a p^{2}}{40000} * H c * F f
$$

Além das características dendrométricas foram também levantados os índices de sobrevivência ao longo do tempo.

Para o ajuste dos valores e projeção de incremento e ganho de área transversal e volume futuro foi utilizado o programa Minitab ${ }^{\circledR}$ (MINITAB, 2015) e testados cinco modelos de equação para melhor explicar a projeção de dados futuros, sendo utilizados os modelos lineares simples, quadrático, logarítmico, hiperbólico, e o modelo não linear monomolecular. Sendo que para modelo não linear monomolecular, foram utilizados os seguintes parâmetros: para theta $A, 60$, para theta $\mathrm{B} 0,3$ e para theta $\mathrm{K} 0$. A tabela 1 demostra os modelos de equações que foram testados.

TABELA 1 Modelos das equações testadas.

\begin{tabular}{lll}
\hline № & Termo & Modelo \\
\hline 1 & Linear simples & DAP $=\mathrm{b}_{0}+\mathrm{b}_{1}$ Idade \\
2 & Quadrático & DAP $=\mathrm{b}_{0}+\mathrm{b}_{1}$ Idade $+\mathrm{b}_{2}$ Idade $^{2}$ \\
3 & Logarítmico & DAP $=\mathrm{b}_{0}+\mathrm{b}_{1} \mathrm{LN}($ Idade $)$ \\
4 & Hiperbólico & DAP $=\mathrm{b}_{0}+\mathrm{b}_{1}(1 /$ ldade $)$ \\
5 & Monomolecular & DAP $=\mathrm{A}(1$-bexp(-kidade $))$ \\
\hline
\end{tabular}

\section{RESULTADOS E DISCUSSÕES}

\section{Levantamento de sobrevivência}

Durante todo o tempo de instalação do experimento foram realizados levantamentos de sobrevivência anualmente, com exceção dos anos de 1998, 2001, 2008, 2011 e 2012, perfazendo assim um total de 23 observações em 28 anos. O índice atual de sobrevivência é de $80 \%$ para plantio de castanha consorciada e $86 \%$ para o plantio com castanha solteira. Para as observações de sobrevivência dos últimos anos, com os indivíduos já adultos, verificou-se que as mortalidades ocorreram devido a ventos fortes ocasionais e também ocorrência de raios. Em estudos realizados na EMBRAPA em Manaus, foram encontrados para um ENCICLOPÉDIA BIOSFERA, Centro Científico Conhecer - Goiânia, v.11 n.22; p. 4612015 
povoamento de 12 anos, um índice de sobrevivência de 78\%, atribuindo as mortalidades também a ventos e raios (COSTA, 2009).

A Tabela 2 e a Figura 3 nos mostram a sobrevivência nos dois sistemas de plantio, tratamento 1 - castanheiras consorciadas e tratamento 2 - castanheiras solteiras, ao longo do tempo.

TABELA 2 Evolução do índice de sobrevivência ao longo de 28 anos de avaliação.

\begin{tabular}{ccccccccccc}
\multicolumn{1}{c}{ Sobrevivência (\%) } & \multicolumn{1}{c}{ Castanha Consorciada } & \multicolumn{6}{c}{ Castanha Solteira } \\
\cline { 2 - 12 } Ano & B1 & B2 & B3 & B4 & Total & B1 & B2 & B3 & B 4 & Total \\
\hline 1988 & 100 & 100 & 100 & 100 & 100 & 100 & 100 & 100 & 100 & 100 \\
1989 & 100 & 100 & 92 & 88 & 95 & 100 & 100 & 100 & 100 & 100 \\
1990 & 100 & 96 & 92 & 84 & 93 & 92 & 100 & 100 & 100 & 98 \\
1991 & 96 & 92 & 92 & 80 & 90 & 84 & 100 & 96 & 100 & 95 \\
1992 & 96 & 92 & 92 & 80 & 90 & 84 & 100 & 96 & 100 & 95 \\
1993 & 96 & 92 & 92 & 80 & 90 & 84 & 100 & 96 & 100 & 95 \\
1994 & 96 & 92 & 92 & 80 & 90 & 84 & 100 & 96 & 100 & 95 \\
1995 & 96 & 92 & 88 & 80 & 89 & 84 & 100 & 96 & 100 & 95 \\
1996 & 96 & 92 & 84 & 80 & 88 & 80 & 100 & 96 & 100 & 94 \\
1997 & 96 & 92 & 84 & 80 & 88 & 80 & 100 & 96 & 100 & 94 \\
1999 & 96 & 92 & 84 & 80 & 88 & 76 & 96 & 96 & 100 & 92 \\
2000 & 92 & 92 & 84 & 80 & 87 & 76 & 96 & 96 & 100 & 92 \\
2002 & 88 & 92 & 84 & 80 & 86 & 76 & 92 & 96 & 100 & 91 \\
2003 & 88 & 92 & 84 & 80 & 86 & 76 & 92 & 96 & 100 & 91 \\
2004 & 88 & 92 & 84 & 80 & 86 & 76 & 92 & 96 & 100 & 91 \\
2005 & 84 & 92 & 84 & 80 & 85 & 76 & 92 & 96 & 100 & 91 \\
2006 & 84 & 92 & 84 & 80 & 85 & 76 & 92 & 96 & 100 & 91 \\
2007 & 84 & 92 & 84 & 80 & 85 & 76 & 92 & 96 & 100 & 91 \\
2009 & 84 & 92 & 80 & 80 & 84 & 76 & 88 & 92 & 100 & 89 \\
2010 & 84 & 92 & 80 & 80 & 84 & 76 & 88 & 92 & 100 & 89 \\
2013 & 84 & 92 & 80 & 80 & 84 & 76 & 88 & 92 & 100 & 89 \\
2014 & 80 & 92 & 80 & 76 & 82 & 76 & 88 & 92 & 100 & 89 \\
2015 & 72 & 92 & 80 & 76 & 80 & 72 & 80 & 92 & 100 & 86 \\
\hline
\end{tabular}




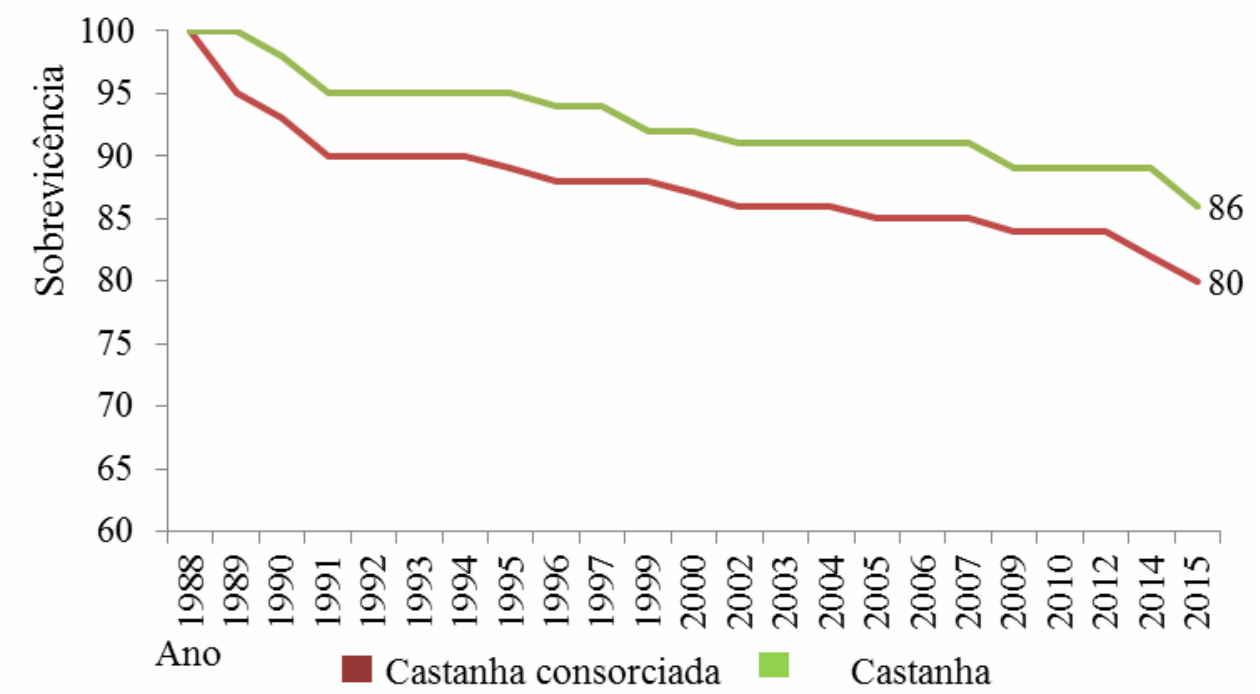

FIGURA 3 Sobrevivência da castanheira-do-brasil em Machadinho d'Oeste, RO.

\section{Levantamento de DAP e área transversal.}

Pelos dados levantados de DAP, conforme a Tabela 3, é possível verificar que a diferença de incremento em DAP é muito pequena entre os tratamentos, para a castanha consorciada aos 28 anos de idade (ano 2015), foram encontrados valores de diâmetro médio na ordem de $59,75 \mathrm{~cm}$, e para o plantio solteiro esses valores ficaram em $60,09 \mathrm{~cm}$.

TABELA-3 Média dos levantamentos de DAP da castanha consorciada e castanha solteira.

\begin{tabular}{|c|c|c|c|c|}
\hline $\begin{array}{l}\text { Castanha } \\
\text { consorciada }\end{array}$ & $\begin{array}{l}\text { Castanha } \\
\text { solteira }\end{array}$ & \multirow[b]{2}{*}{ Ano } & $\begin{array}{c}\text { Castanha } \\
\text { consorciada }\end{array}$ & $\begin{array}{l}\text { Castanha } \\
\text { solteira }\end{array}$ \\
\hline \multicolumn{2}{|c|}{ - } & & \multicolumn{2}{|c|}{$\begin{array}{c}- \\
-\end{array}$} \\
\hline 7,09 & 7,24 & 2003 & 42,68 & 43,02 \\
\hline 11,65 & 11,57 & 2004 & 44,08 & 44,35 \\
\hline 15,40 & 15,29 & 2005 & 47,36 & 47,50 \\
\hline 21,38 & 21,22 & 2006 & 48,76 & 48,75 \\
\hline 24,34 & 24,20 & 2007 & 50,98 & 51,15 \\
\hline 26,99 & 26,87 & 2008 & 52,35 & 52,54 \\
\hline 29,46 & 29,35 & 2009 & 53,72 & 53,94 \\
\hline 31,51 & 31,44 & 2010 & 55,38 & 55,58 \\
\hline 33,52 & 33,52 & 2011 & 56,29 & 56,55 \\
\hline 35,54 & 35,59 & 2012 & 59,11 & 59,38 \\
\hline 37,23 & 37,32 & 2013 & 58,16 & 58,46 \\
\hline 39,10 & 39,33 & 2014 & 57,20 & 57,53 \\
\hline 40,97 & 41,35 & 2015 & 59,75 & 60,09 \\
\hline
\end{tabular}

ENCICLOPÉDIA BIOSFERA, Centro Científico Conhecer - Goiânia, v.11 n.22; p. 4632015 
Após análise estatística para determinar as diferenças entre tratamentos para o incremento em DAP, não foi observada diferença significativa $(p>0,05)$ para o DAP entre os plantios consorciado e solteiro, porém, houve diferença entre os anos (Tabela 4).

TABELA 4 Anova dos DAPs entre castanha consorciada e castanha solteira.

\begin{tabular}{lccccc}
\hline \multicolumn{1}{c}{$\mathrm{FV}$} & $\mathrm{GL}$ & $\mathrm{SQ}$ & $\mathrm{QM}$ & $\mathrm{Fc}$ & $\mathrm{Pr}>\mathrm{Fc}$ \\
\hline Bloco & 3 & 164,20 & 54,73 & 620 & 0,65 \\
Tratamento & 1 & 3,79 & 3,79 & 43 & 0,85 \\
erro 1 & 3 & 264,88 & 88,29 & & \\
Idade & 25 & 48940,86 & 1957,63 & 870979 & 0,00 \\
erro 2 & 75 & 168,57 & 2,25 & & \\
Tratamento*idade & 25 & 7,81 & 0,31 & 307 & 1,00 \\
erro 3 & 75 & 76,21 & 1,02 & & \\
\hline Total corrigido & 207 & 49626,31 & & \\
\hline CV 1 $(\%)=$ & 23,41 & \multicolumn{5}{c}{ Número de 208} \\
CV 2 (\%) = & 3,74 & \multicolumn{5}{c}{ observações } \\
CV 3 (\%) = & 2,51 & & \\
Média geral: & 40,14 &
\end{tabular}

Para o tratamento 1, plantio consorciado, aos 28 anos de idade, o valor de $\mathrm{g}$ ficou em $0,2946 \mathrm{~m}^{2}$, e para o tratamento 2, plantio solteiro o valor encontrado foi de $0,2996 \mathrm{~m}^{2}$ (tabela 5).

TABELA 5 Incremento da área transversal ao longo do tempo em plantio de castanha-do-brasil em Machadinho d'Oeste, RO.

\begin{tabular}{|c|c|c|c|c|c|c|c|c|c|}
\hline \multirow{2}{*}{ Ano } & \multirow{2}{*}{ Idade } & Consorciada & Solteira & Média & \multirow{2}{*}{ Ano } & \multirow{2}{*}{ Idade } & Consorciada & Solteira & Média \\
\hline & & & $\mathrm{g}\left(\mathrm{m}^{2}\right)$ & ---------- & & & \multicolumn{3}{|c|}{ 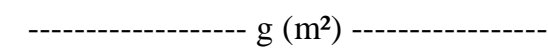 } \\
\hline 1990 & 3 & 0,0046 & 0,0050 & 0,0048 & 2003 & 17 & 0,1484 & 0,1544 & 0,1514 \\
\hline 1991 & 4 & 0,0114 & 0,0112 & 0,0113 & 2004 & 18 & 0,1587 & 0,1634 & 0,1611 \\
\hline 1992 & 5 & 0,0195 & 0,0191 & 0,0193 & 2005 & 19 & 0,1832 & 0,1856 & 0,1844 \\
\hline 1993 & 6 & 0,0373 & 0,0364 & 0,0368 & 2006 & 20 & 0,1946 & 0,1943 & 0,1944 \\
\hline 1994 & 7 & 0,0481 & 0,0470 & 0,0475 & 2007 & 21 & 0,2129 & 0,2165 & 0,2147 \\
\hline 1995 & 8 & 0,0588 & 0,0580 & 0,0584 & 2009 & 23 & 0,2368 & 0,2408 & 0,2388 \\
\hline 1996 & 9 & 0,0701 & 0,0689 & 0,0695 & 2010 & 24 & 0,2514 & 0,2559 & 0,2537 \\
\hline 1997 & 10 & 0,0802 & 0,0796 & 0,0799 & 2012 & 26 & 0,2689 & 0,2750 & 0,2719 \\
\hline 1999 & 12 & 0,1027 & 0,1033 & 0,1030 & 2014 & 27 & 0,2880 & 0,2918 & 0,2899 \\
\hline 2000 & 14 & 0,1125 & 0,1136 & 0,1131 & 2015 & 28 & 0,2946 & 0,2996 & 0,2971 \\
\hline 2002 & 16 & 0,1367 & 0,1429 & 0,1398 & & & & & \\
\hline
\end{tabular}

\section{Volume médio por árvore e volume/ha}

Para a determinação de volume comercial, foi utilizado g média geral, altura comercial geral e índice de fator de forma $(F f)$ de 0,7 , visto que não foi determinado um índice de $\mathrm{Ff}$ específico para esse povoamento, por isso optou-se por utilizar o 
índice de padrão geral. Quanto ao levantamento de volume por ha foi considerado o no atual de indivíduos, mas para a estimativa de potencial de volume foi considerado uma densidade de 69 indivíduos por ha, que corresponde a densidade em um espaçamento entre planta de $12 \mathrm{~m} \times 12 \mathrm{~m}$, que é o utilizado nesse experimento. $O$ resultado encontrado para o volume por árvore foi de 3,89 m³árvore, e de volume por hectare foi de $268,51 \mathrm{~m}^{3} / \mathrm{ha}$. (Tabela 6 ).

TABELA 6 Resumo dos resultados dendrométricos encontrados no plantio de castanha-do-brasil em Machadinho d'Oeste, RO.

\begin{tabular}{llllll}
$\mathrm{g}\left(\mathrm{m}^{2}\right)$ & $\mathrm{G}\left(\mathrm{m}^{2}\right)$ & $\mathrm{Hc}(\mathrm{m})$ & $\mathrm{Ht}(\mathrm{m})$ & $\mathrm{m}^{3} /$ árvore & $\mathrm{m}^{3} / \mathrm{ha}$ \\
\hline
\end{tabular}

Castanha

Consorciada

0,29463

20,3294

18,8

28,6

3,87

267,29

Castanha Solteira

0,29958

20,6711

18,6

28,3

3,91

269,72

Média

0,29710

20,50022

18,7

28,5

3,89

268,52

IMA

0,01061

0,73215

0,7

1,0

0,14

9,59

Em estudos da espécie, a castanheira foi considerada como sendo uma boa opção para plantios sustentáveis em áreas degradadas, levando em consideração que tem boa forma de fuste e desrama natural dos galhos, apresentando crescimento relativamente rápido, tem também relativa rusticidade e tolerância a luz (SOUZA et al, 2008). Em estudos realizados na região de Manaus, com povoamentos de 10 anos, foram encontrados valores de diâmetro médio com 13,9 $\mathrm{cm}$, altura média total de $15,41 \mathrm{~m}$, com volume médio de 117,3 $\mathrm{m}^{3} / \mathrm{há}$ e IMA de $11,73 \mathrm{~m}^{3}$ (FERNANDES \& ALENCAR, 1993). No Pará, na Estação Experimental de Curuá Una, plantios abertos tiveram um índice de sobrevivência de $95 \%$ e IMA em altura de $0,98 \mathrm{~m}$, e IMA em DAP de $1,25 \mathrm{~cm}$, com relação ao volume os dados mostraram um IMA de 17,9 m³/ha/ano (ÁVILA, 2006, citado por SOUZA et al 2008). No município de Cantá - Roraima, em experimento de SAF com castanha-do-brasil aos 10 anos, foram encontrados valores médios de $25,5 \mathrm{~cm}$ de DAP, altura total de 14,0 m, e índice de sobrevivência de 98,6\% (FERREIRA, 2009). Outros dados de crescimento de castanheiras foram obtidos em estudos na EMBRAPA, em Manaus em SAF com 12 anos, onde levantou-se dados de DAP com $37,9 \mathrm{~cm}$, altura total média de 20,9 m e índice de sobrevivência de $78 \%$, onde as mortalidades ocorreram devido a ventos e raios (COSTA, 2009). A Tabela 7 demonstra o desempenho de alguns plantios de castanha do Brasil em diferentes localidades.

TABELA 7 Comparativo de crescimento em alguns plantios com idades próximas.

\begin{tabular}{|c|c|c|c|c|c|}
\hline Local & $\begin{array}{l}\text { Idade } \\
\text { (anos) }\end{array}$ & DAP (cm) & $\begin{array}{l}\mathrm{Ht}(\mathrm{m}) \\
-\mathrm{IMA}-\end{array}$ & $\operatorname{Vol}\left(m^{3}\right)$ & $\begin{array}{c}\text { Sobrevivência } \\
\%\end{array}$ \\
\hline Cantá - RR (FERREIRA, 2009) & 10 & 2,55 & 1,40 & -- & 98 \\
\hline Curua Una - PA (ÁVILA, 2006) & -- & 1,25 & 0,98 & 17,9 & 95 \\
\hline Machadinho - RO & 10 & 3,14 & 2,18 & -- & 83 \\
\hline Manacapuru - AM (SOARES, 2003) & 10 & 3,10 & 1,60 & -- & 57 \\
\hline Manaus - AM (FERNANDES, 1993) & 10 & 1,39 & 1,54 & 11,73 & 69 \\
\hline $\begin{array}{l}\text { Manaus - AM (EMBRAPA)(COSTA, } \\
\text { 2009) }\end{array}$ & 12 & 3,16 & 1,74 & -- & 78 \\
\hline
\end{tabular}

Adaptação feita pelos autores. 
Considerando as condições edafoclimáticas, com distribuição irregular das chuvas, e um período seco entre abril e agosto, juntamente com as características de solo com alta acidez, altos níveis de alumínio, e baixos níveis dos demais elementos como fosforo $(\mathrm{P})$, potássio $(\mathrm{K})$, cálcio $(\mathrm{Ca})$ e magnésio $(\mathrm{Mg})$, essenciais ao desenvolvimento dos vegetais, pode-se considerar que a castanha teve um bom crescimento, e que não ocorreu diferenças significativas entre as parcelas consorciadas daquelas em plantios solteiros.

Para efeito de comparação com outros plantios utilizou-se dados de alguns plantios com dez anos de idade ou próximo disso, com os do experimento nessa mesma idade, visto que não foram encontrados dados dos povoamentos com a idade atual. Essa comparação teve como objetivo verificar o desempenho do plantio frente a outros plantios. Ao se comparar esses dados, observou-se que em termos de incremento, os números encontrados superam praticamente todos aqueles encontrados nos outros plantios, para o DAP obteve-se valor de IMA de $3,14 \mathrm{~cm}$, ficando pouco abaixo de Manaus (EMBRAPA) com 3,16 cm, e acima do IMA encontrado em Uruá Una (PA) com 1,25 cm. Quando compara-se o incremento em altura, esse supera todos os outros, com IMA de 2,18 m, bem superior ao menor, que ocorreu em Curuá Una (PA) com 0,98 m. Os índices de sobrevivência ficaram em níveis intermediário com $83 \%$, onde o maior desempenho foi em Cantá (RR) com 98\%, e o menor em Manacapuru (AM) com 57\%.

Com os levantamentos dendrométricos atuais foram encontrados dados de volume comercial para média geral do plantio na ordem de 3,91 m³/árvore, com um IMA de $9,59 \mathrm{~m} 3 / \mathrm{ha} /$ ano. Para a altura total foi encontrado IMA de 1,02 m, para o DAP $2,15 \mathrm{~cm}$ e para área transversal $0,2971 \mathrm{~m}^{2}$ (Figura 4).

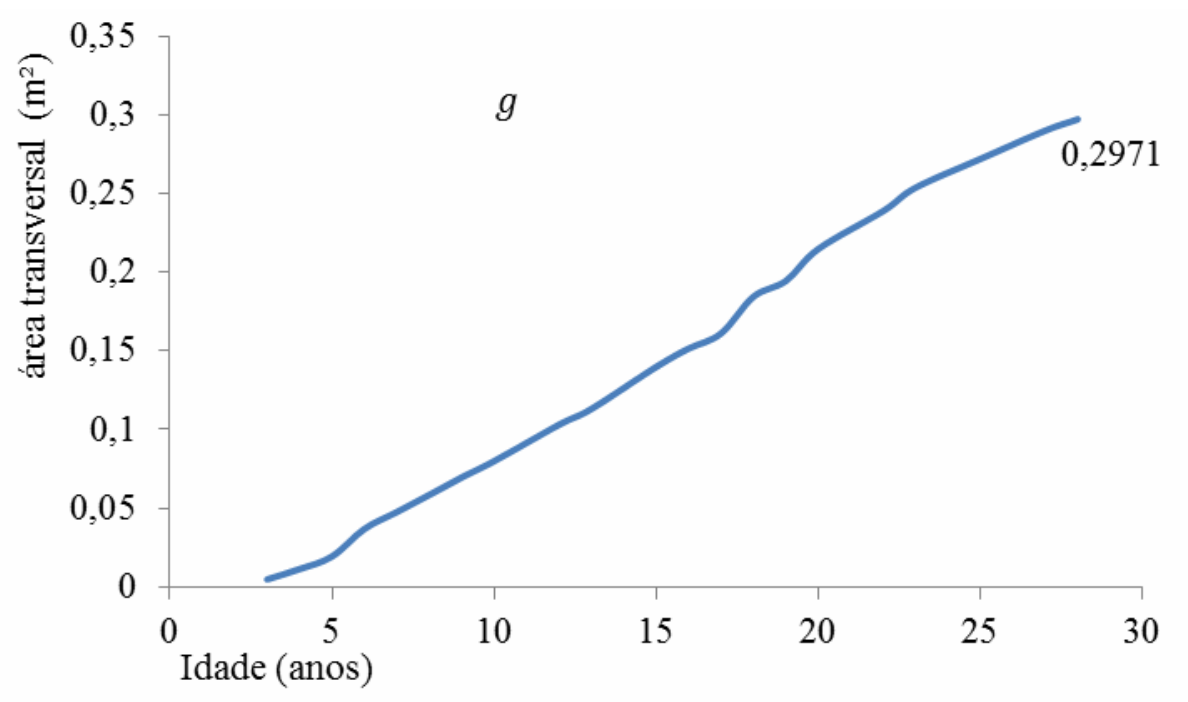

FIGURA 4 Incremento médio anual (IMA) da área transversal.

Pela análise da figura 4 que mostra crescimento em área transversal, pode-se dizer que o povoamento do experimento de Machadinho do Oeste com idade de 28 anos, ainda está com a curva em ascendência sem sinais de estabilização, podendo-se inferir que para essa situação o povoamento encontra-se em desenvolvimento e ainda não atingiu seu ponto de estagnação, se considerar as condições adversas de fertilidade de solo para o desenvolvimento das plantas, e o desenvolvimento do povoamento pode ser considerado satisfatório. 


\section{Estimativa de IMA, ICA e volume futuro.}

Foi determinado por estimativa o ganho de volume, incremento médio anual (IMA) e incremento corrente anual (ICA) até os 40 anos de idade. Para projeção foi utilizado o DAP médio e a altura comercial média de toda população. A curva de projeção no gráfico de voluma (Figura 6) se inicia a partir do $23^{\circ}$ ano, idade em que foi tomada a altura comercial com hipsômetro eletrônico, onde a confiabilidade dos dados é maior.

Após os testes, as fórmulas foram ajustadas e verificado o melhor modelo, sendo que para projeção de ganhos futuros, foi considerado como melhor modelo aquele com menor quadrado médio do erro (QME). A tabela 8 demostra as fórmulas ajustadas, com seus respectivos valores encontrados.

A equação que matematicamente melhor demostra a projeção de ganho futuro foi a equação $n^{\circ}$ 5, não linear, monomolecular. Através dela foi possível construir a tabela e os gráficos de ganhos futuros. A tabela 10 mostra os dados projetados para o povoamento aos 40 anos de idade.

TABELA 8 Fórmulas ajustadas para calculo das projeções de incremento.

\begin{tabular}{llcc}
\hline $\mathrm{N}^{\circ}$ & Fórmula ajustada & $\mathrm{R}^{2}$ & $\mathrm{QME}$ \\
\hline 1 & $\mathrm{DAP}=8,414+2,040$ Idade & $96,1 \%$ & 10,30 \\
2 & $\mathrm{DAP}=-0,948+3,688$ Idade $-0,05424$ Idade $\wedge 2$ & $99,4 \%$ & 1,58 \\
3 & $\mathrm{DAP}=-23,55+24,633 \mathrm{LNld}$ & 98,54 & 2,94 \\
& & $\%$ & \\
4 & $\mathrm{DAP}=57,54-189,61 / \mathrm{ld}$ & 74,27 & 41,82 \\
& & $\%$ & \\
5 & $\mathrm{DAP}=76,1101 *(1-1,05341 * \exp (-0,0575932 *$ & - & 1,0755 \\
& Idade $)$
\end{tabular}

Através dos valores de DAP corrigidos com os diversos modelos matemáticos, verificou-se que o modelo monomolecular é o que melhor descreve a projeção de crescimento da floresta e também o comportamento do IMA e ICA ao longo de 40 anos. Através da determinação da área transversal corrigida, os resultados obtidos demonstram aumento do IMA até o ponto máximo, atingido aos 24 anos com valor de $0,01032 \mathrm{~m}^{2}$, ocorrendo após essa idade um declínio chegando aos 40 anos com um valor de 0,00914. Para o comportamento do ICA, observou-se que seu ponto máximo se deu aos 12 anos de idade com valor em $0,01317 \mathrm{~m}^{2}$, com declínio até os 40 anos, chegando a $0,00503 \mathrm{~m}^{2}$ (Figura 5). 


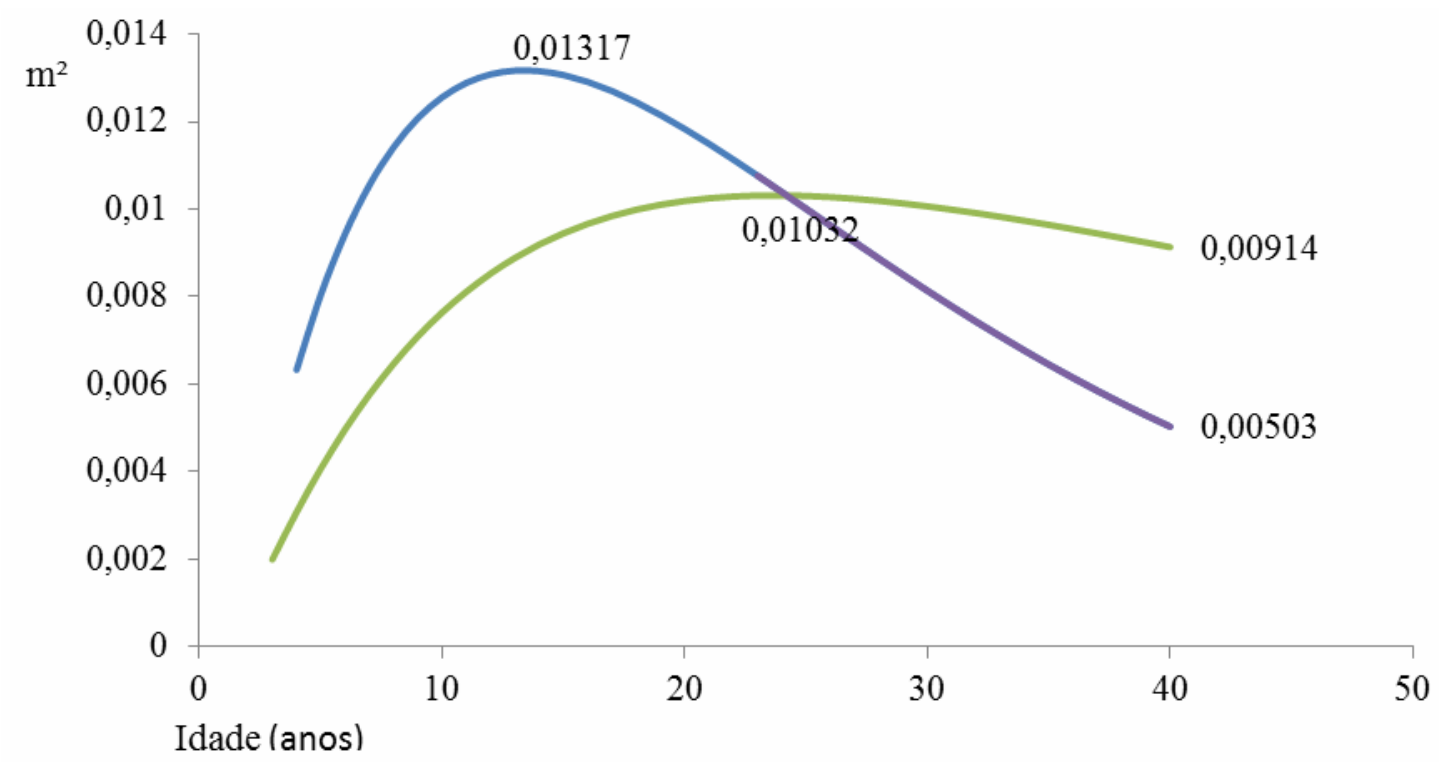

FIGURA 5 Projeção da curva de ICA e IMA para área transversal em plantio de castanha-do-brasil em Machadinho d'Oeste, RO.

TABELA 9 Valores encontrados corrigidos para a idade de 40 anos.

\begin{tabular}{ccccc} 
DAP ajustado & $\mathrm{g}\left(\mathrm{m}^{2}\right)$ & $\mathrm{ICA}\left(\mathrm{m}^{3} / \mathrm{ha} / \mathrm{ano}\right)$ & $\mathrm{IMA}\left(\mathrm{m}^{3} / \mathrm{ha} / \mathrm{ano}\right)$ & Volume $\left(\mathrm{m}^{3}\right)$ \\
\hline 68,21 & 0,3654 & 0,00914 & 0,00503 & 4,81
\end{tabular}

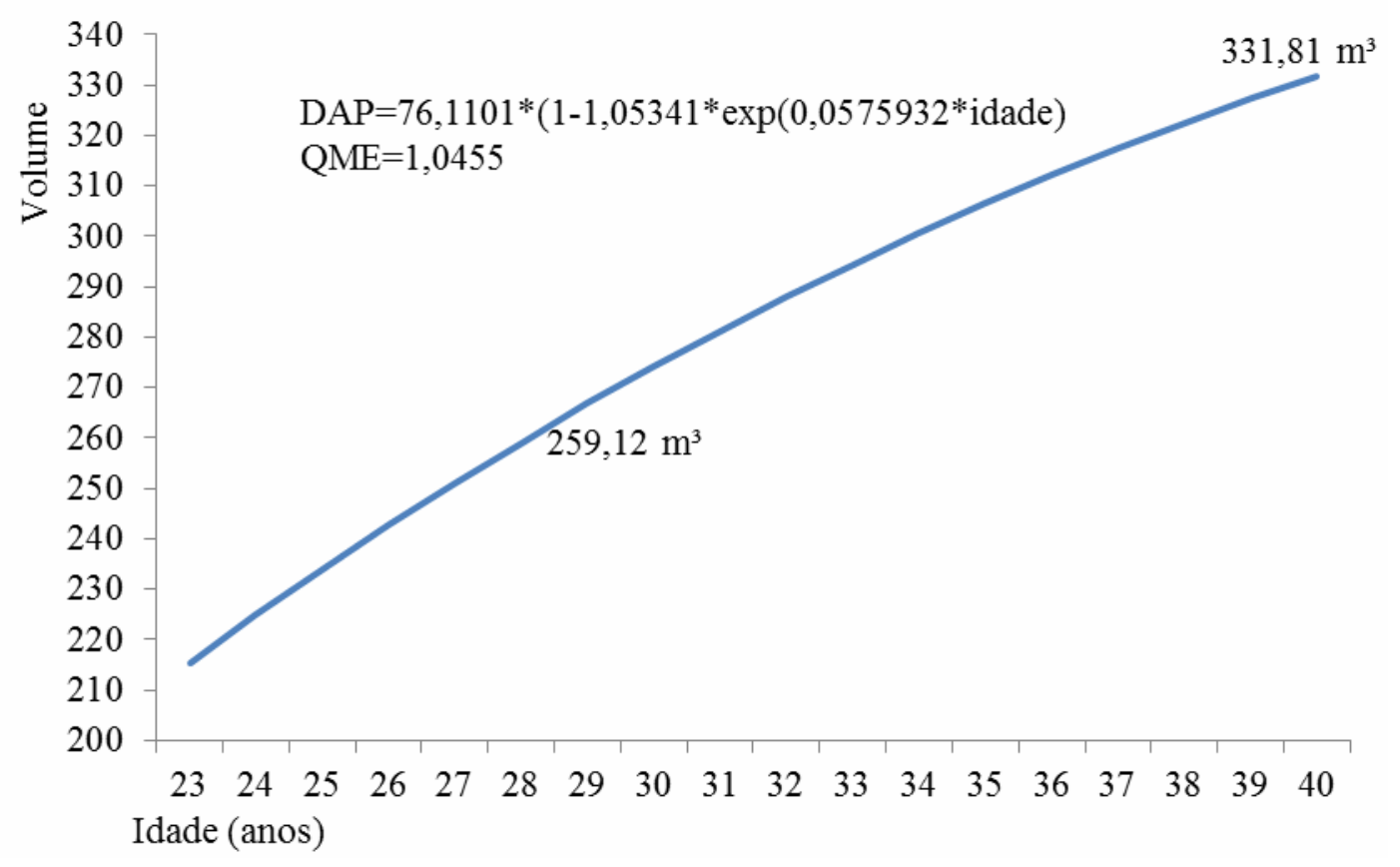

FIGURA 6 Incremento de volume projetado até 40 anos, utilizando a equação ajustada em plantio de castanha-do-brasil, em Machadinho d'Oeste, RO. 
Para montagem da curva de incremento em volume considerou-se uma população de 69 indivíduos por há, com um espaçamento de $12 \mathrm{~m} \times 12 \mathrm{~m}$, que é o utilizado no experimento. Após os ajustes das equações para a projeção do crescimento em volume, obteve-se aos 40 anos $331,81 \mathrm{~m}^{3} /$ ha. (Figura 6).

Os dados de incremento mostram um declínio ao projetarmos os ganhos para além dos 28 anos até a idade de 40 anos, até 28 anos pelas medições realizadas temos um IMA em volume de $9,59 \mathrm{~m}^{3} /$ há/ano, quando projetamos o IMA para 40 anos temos o valor de $8,29 \mathrm{~m}^{3} / \mathrm{ha}$ /ano para essa idade. A redução do ganho de volume após uma certa idade pode estar relacionada com a estabilidade no incremento em altura do povoamento, a partir dessa fase o ganho em volume está mais relacionado ao incremento em DAP.

Pelos estudos das condições de sítio do povoamento, onde temos baixa pluviosidade durante alguns meses do ano, associado a baixa fertilidade do solo, pode ser explicado em parte o pico máximo do IMA em área transversal aos 24 anos de idade e a partir daí seu declínio, considerando que a espécie é de longevidade muito grande, ate 500 anos de sobrevivência, e com diâmetros que chegam a 2,0 m (WADT, 2005), pode-se supor que a idade de declínio poderia ser muito além dos 24 anos. Outra possibilidade seria uma condição biológica natural da espécie, quando atingido essa idade, pela sua própria biologia entraria num estágio de declínio em relação ao IMA, onde essa situação poderia ser considerada normal, mas fica difícil fazer tal afirmação uma vez que não foi encontrado na literatura referências de acompanhamentos dendrométricos que demonstrem as curvas de crescimento com determinação das idades onde ocorre o declínio do IMA, ou ainda a curva de estabilidade do ganho de volume ao longo do tempo em um espaço de 40 anos.

\section{CONCLUSÕES}

Sem utilização de adubação complementar a prognose de volume foi de 331,81 $\mathrm{m}^{3} /$ há, aos 40 anos, demostrando dessa forma que a castanha do brasil (Bertholletia excelsa Bonpl.) é uma espécie que se desenvolve bem em solos de baixa fertilidade sendo portanto indicada para plantios em áreas degradadas, como elemento da recomposição florestal.

\section{REFERÊNCIAS}

BAYMA, M.M.A; et al.; Aspectos da cadeia produtiva da castanha-do-brasil no estado do Acre, Brasil. Boletim Museu Paraense Emílio Goeldi. Ciências Naturais, Belém, v. 9, n. 2, p. 417-426, maio-ago. 2014.

COSTA, J.R.; CASTRO, A.B.C.; WANDELLI, E.V.; CORAL, S.C.T.; SOUZA, S.A.G.; Aspectos silviculturais da castanha-do-brasil (Bertholletia excelsa) em sistemas agroflorestais na Amazônia Central. Acta Amazonica, v. 39(4) p 843 - 850.2009.

FERNANDES, N.P.; ALENCAR, J. da C. Desenvolvimento de árvores nativas em ensaios de espécie 4 castanha do brasil (Bertholletia excelsa H.B.K) dez anos após plantio. Acta Amazônia vol. 23, p 191-198, 1993. 
FERREIRA, L.M.M.; TONINI, H. Comportamento da castanha-do-brasil (Bertholletia excelsa) e da cupiúba (Goupia glabra) em sistema agrosilvicultural na região da Confiança, Cantá - Roraima. Acta Amazônia , v. 39, p 835 - 842, 2009.

HOMMA, A. K. O. (Ed.). Extrativismo vegetal na Amazônia: história, ecologia, economia e domesticação. Brasília, DF: Embrapa, 2014. Cap. 12, p. 193-199.2014.

HOMMA, A.K.O.; MENEZES, A.J.E.A.; MAUÉS, M.M.; Castanheira-do-pará: os desafios do extrativismo para plantios agrícolas. Boletim Museu Paraense Emílio Goeldi. Ciências Naturais, Belém, v. 9, n. 2, p. 293-306, maio/ago. 2014.

INMET Instituto Nacional de Meteorologia, Dados Históricos. Disponível em: <www.inmet.gov.br> Acesso em: 29 abril 2015.

MINITAB. Minitab for Windows Release 17. State College: Minitab Inc., 2015

NUNES, P.C.; VIVAN, J.L. Florestas, sistemas agroflorestais e seus serviços ambientais e econômicos em Juruena-MT. Ed 1 Associação de Desenvolvimento Rural de Juruena. Projeto Poço de Carbono Juruena / ADERJUR-Juruena. Cuiabá 2011, 42p. ISBN: 978-85-912647-0-4

SABOGAL, C. et al; Sivicultura na amazônia brasileira: avaliação de experiências e recomendações para implementação e melhoria dos sistemas. Belém CIFOR - 2006. 190 p ISBN 979-24-4644-3, CDD 634-928

SILVA, M. F. da; SILVA, L. M. da; BATISTA, K. D.; WADT, L. H. de O. Correlação entre teores de nutrientes do solo, foliar e produção da castanha-do-brasil na Amazônia Sul Ocidental. II Reunião de Ciência Do Solo da Amazônia Ocidental, 2014, Porto Velho. Anais. Porto Velho: SBCS, 2014.

SILVA, V. S.; MARTINS, K.; CAMPOS, T. DE WADT, L. H. de O. Sistema reprodutivo de Bertholletia excelsa e implicações para o manejo e conservação da espécie. II Congresso Brasileiro de Recursos Genéticos. 2012, Belém, PA. Anais... Brasília, DF: Sociedade Brasileira de Recursos Genéticos, 2012

SOUZA, C.R. de; et al. Castanha do brasil (Bertholletia excelsa Humb. \& Ponbl.). Manuas: Embrapa Amazonia Ocidental. 2008. 22p. ISBN 1517-3535. 